Cooperative Oscillatory Behavior of Mutually Coupled Dynamical Systems

Alexander Pogromsky and Henk Nijmeijer

WFW report 98.044

A. Pogromsky, H. NiJMeijeR

Faculty of Mechanical Engineering

Eindhoven University of Technology

December 1998 


\title{
Cooperative oscillatory behavior of mutually coupled dynamical systems
}

\author{
A. Yu. Pogromsky* \\ Institute for Problems of Mechanical Engineering \\ 61, Bolshoy V.O., St.Petersburg, 199178, RUSSIA \\ sasha@ccs.ipme.ru \\ H. Nijmeijer ${ }^{\dagger}$ \\ Faculty of Mathematical Sciences \\ University of Twente, PO Box 217, \\ 7500 AE Enschede, The Netherlands \\ h.nijmeijer@math.utwente.nl
}

\begin{abstract}
In this paper we make a qualitative study of the dynamics of diffusively coupled identical systems. In particular, we derive conditions on the mutual coupling of the systems that guarantee global synchronization of the systems, and analougously, conditions that guarantee certain oscillatory behavior to occur.

Keywords: Synchronization, Cellular neural networks, passivity.

\section{Introduction}

Recently an increasing interest has been devoted to the study of cellular neural networks (CNNs) $[4,22,23,19]$. A CNN consists of mutually coupled dynamical systems and due to interactions it can demonstrate very complex behavior even in case when each cell itself is described by simple equations. Among the possible applications of CNNs we mention the very interesting fields of telecommunication [12] and mathematical biology [9].

Even CNNs consisting of simple cells form, after coupling, high dimensional nonlinear systems, and therefore such CNNs are difficult to study analytically. However, recently some progress in the qualitative study of CNNs has been made. A thorough analysis of

*The work was performed while the author was with the Faculty of Mechanical Engineering, Eindhoven Technical University, The Netherlands

${ }^{\dagger}$ Also at Faculty of Mechanical Engineering, Technical University of Eindhoven, PO Box 513, 5600 MB, Eindhoven, The Netherlands.
\end{abstract}


CNNs consisting of cells described by first order equations coupled in linear 1-D arrays can be found in $[23,19]$. It was shown that even in such simple setting it is possible to observe nontrivial phenomena called local diffusion and global propagation.

In the present paper we are interested in oscillatory behavior in networks composed of identical dynamical systems and coupled in arbitrary arrays via diffusive coupling, that is the systems are mutually linearly coupled with some restrictions on the coupling matrix. Two particular phenomena will be studied: synchronization and generation of oscillations via diffusion. We will present conditions in terms of the coupling that guarantee global synchronization in the diffusive network and we will show that in growing networks (the number of cells grows) when the number of interconnections grows no more than linearly with respect to the number of cells the systems eventually will lose this synchronization property. However the synchronization can be retained if one allows for a quadratic growth of the number of interconnections. Our research generalizes some studies of synchronization in arrays of linearly coupled dynamical systems (see e.g.[26, 1]) because we allow for an arbitrary topology of interconnections.

We will also show that diffusive networks can generate oscillations which can be observed for almost all initial conditions even in case when each unconnected cell is globally asymptotically stable. For diffusive coupling this phenomenon is possible only if each cell is described by a third (or larger) order differential equation.

The paper is organized as follows. First (Sections 2 and 3) we present necessary background material. A notion of diffusive coupling will be defined in Section 4 . Section 5 deals with the synchronization phenomena occuring via diffusion. In Section 6 we present condition resulting in oscillatory behavior in diffusively coupled systems. Some useful properties of diffusive networks are discussed in Section 7. Section 8 contains some concluding remarks.

\section{Notations}

The Euclidean norm in $\mathbb{R}^{n}$ is denoted simply as $|\cdot|,|x|^{2}=x^{\top} x$, where ${ }^{\top}$ defines transposition. We will study notions relative to nonempty subsets $\mathcal{A}$, of $\mathbb{R}^{n}, 0 \in \mathcal{A}$; for such a set $\mathcal{A},|x|_{\mathcal{A}}=\operatorname{dist}(x, \mathcal{A})=\inf _{\eta \in \mathcal{A}} \operatorname{dist}(x, \eta)$ denotes the Hausdorf distance from $x \in \mathbb{R}^{n}$ to $\mathcal{A}$.

A function $V: X \rightarrow \mathbb{R}_{+}$defined on a subset $X$ of $\mathbb{R}^{n}, 0 \in X$ is positive definite if $V(x)>0$ for all $x \in X \backslash\{0\}$ and $V(0)=0$. It is radially unbounded (if $X=\mathbb{R}^{n}$ ) or proper if $V(x) \rightarrow \infty$ as $|x| \rightarrow \infty$. A nonnegative function $V: X \rightarrow \mathbb{R}_{+}$is said to be positive definite with respect to the set $\mathcal{A}$ if $V(x)>0$ for all $x \in X \backslash \mathcal{A}$ and $V(x)=0$ for all $x \in \mathcal{A}$. It is proper with respect to $\mathcal{A}$ if boundedness of $V(x)$ implies boundedness of $|x|_{\mathcal{A}}$.

If a quadratic form $x^{\top} P x$ with a symmetric matrix $P=P^{\top}$ is positive definite then the matrix $P$ is called positive definite. For positive definite matrices we use the notation $P>0$; moreover $P>Q$ means that the matrix $P-Q$ is positive definite.

A matrix $A$ for which all eigenvalues have negative real parts is called Hurwitz or stable.

An invariant set $\mathcal{A} \subset \mathbb{R}^{n}$ for the dynamics $\dot{x}=f(x)$ is said to be noncritically stable if it is Lyapunov stable, that is all solutions starting close enough to $\mathcal{A}$ remain close to $\mathcal{A}$ for all $t$ and additionally $|x(t)|_{\mathcal{A}} \leq C e^{-\delta t}|x(0)|_{\mathcal{A}}$ for sufficiently small $|x(0)|_{\mathcal{A}}$ with $C, \delta$ 
perhaps depending on $|x(0)|_{\mathcal{A}}$.

The system $\dot{x}=f(x)$ is called Lagrange stable if all its solutions are bounded. If the ultimate bounds can be chosen independently of the initial conditions we will say that all solutions are ultimately bounded.

For matrices $A$ and $B$ the notation $A \otimes B$ (the Kronecker product) stands for the matrix composed of submatrices $A_{i j} B$, i.e.

$$
A \otimes B=\left(\begin{array}{cccc}
A_{11} B & A_{12} B & \cdots & A_{1 n} B \\
A_{21} B & A_{22} B & \cdots & A_{2 n} B \\
\vdots & \vdots & \ddots & \vdots \\
A_{n 1} B & A_{n 2} B & \cdots & A_{n n} B
\end{array}\right)
$$

where $A_{i j}, i, j=1 \ldots n$, stands for the $i j$-th entry of the $n \times n$ matrix $A$.

\section{On passivity with respect to sets}

Consider the nonlinear time-invariant affine system:

$$
\left\{\begin{array}{l}
\dot{x}=f(x)+g(x) u \\
y=h(x)
\end{array}\right.
$$

where $x \in \mathbb{R}^{n}$ is the state, $u \in \mathbb{R}^{m}$ is the input which is assumed to be a continuous and (essentially) bounded function of time: $u(\cdot) \in \mathcal{C}^{0} \cap \mathcal{L}_{\infty}, y(t) \in \mathbb{R}^{m}$ is the output; $f: \mathbb{R}^{n} \rightarrow \mathbb{R}^{n}, f(0)=0, g: \mathbb{R}^{n} \rightarrow \mathbb{R}^{n \times m}$ are smooth enough to ensure existence of solutions in any reasonable sense, e.g. in the sense of Filippov, at least on a finite time interval $0<t<T_{x_{0}, u} ; h: \mathbb{R}^{n} \rightarrow \mathbb{R}^{m}$ is the output mapping.

Suppose there exist a nonnegative differentiable ${ }^{1}$ storage function $V: \mathbb{R}^{n} \rightarrow \mathbb{R}_{+}, V(0)=$ 0 and nonnegative continuous function $S: \mathbb{R}^{n} \rightarrow \mathbb{R}_{+}, S(0)=0$ such that for all admissible inputs $u$ and initial conditions $x(0)=x_{0}$ and for all time instants $0 \leq t<T_{x_{0}, u}$ the following dissipation inequality is valid:

$$
\dot{V}(x, u) \leq y^{\top} u-S(x) .
$$

Then the system (2) is called a passive system, see, e.g. [10,3]. If, additionally, $S$ is positive definite then the system (2) is called strictly passive.

From the definition of passive systems one can draw two important conclusions. First, if the strictly passive system (2) possesses an inherent dynamics consistent with the constraint $y=0$ (zero dynamics) and $V$ is positive definite, then the origin is an asymptotically stable equilibrium of the zero dynamics. Secondly, if $V$ is positive definite, then the origin is a stable equilibrium of the free system $(u \equiv 0$ ) (in case of strict passivity the origin is asymptotically stable).

\footnotetext{
${ }^{1}$ In fact, the function $V$ is not necessarily differentiable. In this case we will assume that $V$ is locally Lipschitz continuous (it satisfies a Lipschitz condition on any compact set). Indeed, if $x(t)$ is a bounded solution in the sense of Filippov, then it is absolutely continuous function of time and therefore $V(x(t))$ is also an absolutely continuous function of time, that is its time derivative exists almost everywhere.
} 
The theory of passive systems plays an important role in modern control theory ([3, $13,25])$. In a natural way it extends the notion of positive real linear systems to nonlinear systems.

In this paper we need some weakened version of passivity because we focus our attention on systems exhibiting oscillatory behavior for $u \equiv 0$.

Next we define a semipassive system. This notion was introduced in [15]; in [17] an equivalent notion was called quasipassivity. Roughly speaking a semipassive system behaves as a passive system for sufficiently large $|x|$. More precisely, assume that there exists a nonnegative function $V: \mathbb{R}^{n} \rightarrow \mathbb{R}_{+}$such that for all admissible inputs, for all initial conditions and for all $t$ for which the corresponding solution of (2) exists we have

$$
\dot{V}(x, u) \leq y^{\top} u-H(x)
$$

where the function $H: \mathbb{R}^{n} \rightarrow \mathbb{R}$ is nonnegative outside some ball:

$$
\exists \rho>0, \forall|x| \geq \rho \Longrightarrow H(x) \geq \varrho(|x|)
$$

for some continuous nonnegative function $\varrho$ defined for $|x| \geq \rho$. If the function $H$ is positive outside some ball, i.e. (5) holds for some continuous positive function $\varrho$, then the system (2) is said to be strictly semipassive.

The notions of semipassivity and strict semipassivity can be treated as a generalization of the passivity concept to the passivity with respect to compact sets. Indeed, if $V$ is radially unbounded then from (4) it follows that semipassivity of (2) implies that (2) is Lagrange stable for $u \equiv 0$ or all solutions of (2) are ultimately bounded for $u \equiv 0$ (in case of strict semipassivity). However, the concept of passivity with respect to sets can be introduced in a slightly different manner [15]. Namely, if the function $V$ in (3) obeys $V(x)=0$ for all $x \in \mathcal{A}$ and the function $S$ is positive definite with respect to $\mathcal{A}$ then the system (2) is referred to as strictly passive with respect to $\mathcal{A}$. From the notion of strict semipassivity with respect to sets it is also possible to derive a stability property of the set $\mathcal{A}$ of free system $(u \equiv 0)$. Indeed, if $V$ is proper w.r.t $\mathcal{A}$ and $\mathcal{A}$ is compact, then $\mathcal{A}$ is globally asymptotically stable set for (2). Moreover, if $\mathcal{A}$ is not compact but all solution of the free system exist on the infinite time interval, then again the set $\mathcal{A}$ is asymptotically stable set for (2) with $u \equiv 0$. These statements immediately follow from Lyapunov theorems for stability of sets, see e.g. [11].

In fact, there is a close connection between the concepts of semipassivity and passivity with respect to compact sets, however, we will not discuss this topic here.

The concept of semipassivity alows one to find simple conditions which ensure boundedness of the solutions of interconnected systems. Consider $k$ identical systems of the form (2):

$$
\left\{\begin{array}{l}
\dot{x}_{j}=f_{j}\left(x_{j}\right)+g_{j}\left(x_{j}\right) u_{j} \\
y_{j}=h_{j}\left(x_{j}\right)
\end{array}\right.
$$

where $j=1, \ldots, k$. 
Define the symmetric $k \times k$ matrix $\Gamma$ as

$$
\Gamma=\left(\begin{array}{ccccc}
\sum_{i=1}^{k} \gamma_{1 i} & -\gamma_{12} & -\gamma_{13} & \cdots & -\gamma_{1 k} \\
-\gamma_{21} & \sum_{i=1}^{k} \gamma_{2 i} & -\gamma_{23} & \cdots & -\gamma_{2 k} \\
\vdots & \vdots & \vdots & \vdots & \vdots \\
-\gamma_{k 1} & -\gamma_{k 2} & -\gamma_{k 3} & \cdots & \sum_{i=1}^{k} \gamma_{k i}
\end{array}\right)
$$

where $\gamma_{i j}=\gamma_{j i} \geq 0$. The matrix $\Gamma$ is symmetric and therefore all its eigenvalues are real. Moreover applying Gerschgorin's theorem about localization of eigenvalues (see, e.g. [21]) one can see that all eigenvalues of $\Gamma$ are nonnegative, that is the matrix $\Gamma$ is positive semidefinite.

The following result gives conditions under which the solutions of the interconnected systems (6) are bounded.

Lemma 1 ([16]) Consider the systems (6) in closed loop with the following feedback

$$
u_{j}=-\gamma_{j 1}\left(\tilde{y}_{j}-\tilde{y}_{1}\right)-\gamma_{j 2}\left(\tilde{y}_{j}-\tilde{y}_{2}\right)-\ldots-\gamma_{j k}\left(\tilde{y}_{j}-\tilde{y}_{k}\right)
$$

where $\tilde{y}_{j}=\tilde{h}_{j}\left(x_{j}\right)$ and $\tilde{h}_{j}: \mathbb{R}^{n_{j}} \rightarrow \mathbb{R}^{m}$ is a smooth mapping such that $\tilde{h}_{j}(0)=0$. Suppose that the systems (6) are semipassive with respect to input $u_{j}$ and output $y_{j}=h_{j}\left(x_{j}\right)$ with radially unbounded storage functions $V_{j}: \mathbb{R}^{n_{j}} \rightarrow \mathbb{R}_{+}$and functions $H_{j}$ satisfying (5), $j=1, \ldots, k$. Assume the function

$$
H(x)=\sum_{j=1}^{k} H_{j}\left(x_{j}\right)+y^{\top}\left(I_{m} \otimes \Gamma\right) \tilde{y}
$$

with $x=\operatorname{col}\left(x_{1}, \ldots, x_{k}\right), y=\operatorname{col}\left(y_{1}, \ldots, y_{k}\right), \tilde{y}=\operatorname{col}\left(\tilde{y}_{1}, \ldots, \tilde{y}_{k}\right)$, satisfies

$$
\exists \rho>0, \forall|x| \geq \rho \Longrightarrow H(x) \geq \varrho(|x|)
$$

for some continuous function $\varrho$ defined for $|x| \geq \rho$. Then if the function $\varrho$ is nonnegative, then all solutions of the closed loop system (6), (8) exist for all $t \geq 0$ and are bounded, that is the system (6), (8) is Lagrange stable, moreover if the function $\varrho$ is positive, then the closed loop system (6), (8) has ultimately bounded solutions.

Corollary 1 ([16]) Suppose that the systems (6) are semipassive with radially unbounded storage functions $V_{j}: \mathbb{R}^{n_{j}} \rightarrow \mathbb{R}_{+}$. Then all solutions of the systems (6) in closed loop with the feedback

$$
u_{j}=-\gamma_{j 1}\left(y_{j}-y_{1}\right)-\gamma_{j 2}\left(y_{j}-y_{2}\right)-\ldots-\gamma_{j k}\left(y_{j}-y_{k}\right)
$$

with $\gamma_{j i}=\gamma_{i j} \geq 0$, exist for all $t \geq 0$ and are bounded, that is, the system (6), (10) is Lagrange stable. Moreover, if the systems (6) are strictly semipassive with radially unbounded storage functions $V_{j}: \mathbb{R}^{n_{j}} \rightarrow \mathbb{R}_{+}$then all solutions of the coupled system (6), (10) exist for all $t \geq 0$ and are ultimately bounded. 


\section{Diffusively coupled dynamical systems}

Let us give a definition of diffusive coupling of $k$ identical systems. This definition was introduced in [16] and was inspired by the paper of Smale on interaction between two cells [20]. We will understand a diffusive medium as a system of ordinary differential equations consisting of interconnected identical systems. Each separate system has an input and output of the same dimension. The diffusive coupling is described by a static relation between all inputs and all outputs. Notice that our approach to describe the diffusion is different from that proposed in [23].

Definition 1 Given the systems

$$
\left\{\begin{array}{l}
\dot{x}_{j}=f\left(x_{j}\right)+B u_{j} \\
y_{j}=C x_{j}
\end{array}\right.
$$

where $j=1, \ldots, k, x_{j}(t) \in \mathbb{R}^{n}$ is the state of the $j$-th system, $u_{j}(t) \in \mathbb{R}^{m}$ is the input, $y_{j}(t) \in \mathbb{R}^{m}$ is the output of the $j$-th system, $f(0)=0$ and $B, C$ are constant matrices of appropriate dimension. We say that the systems (11) are diffusively coupled if the matrix $C B$ is similar to a positive definite matrix and the systems (11) are interconnected by the following feedback

$$
u_{j}=-\gamma_{j 1}\left(y_{j}-y_{1}\right)-\gamma_{j 2}\left(y_{j}-y_{2}\right)-\ldots-\gamma_{j k}\left(y_{j}-y_{k}\right)
$$

where $\gamma_{i j}=\gamma_{j i} \geq 0$ are constants such that $\sum_{j \neq i}^{k} \gamma_{j i}>0$ for all $i=1, \ldots, k$.

A motivation for this definition comes from the paper by Smale [20] who studied oscillatory behavior of coupled cells when each unconnected cell is globally asymptotically stable. This phenomenon will be briefly described in Section 6 , for more details, see [16].

\section{Synchronization of diffusively coupled systems}

Nowadays synchronization of dynamical systems is a very popular topic. It attracts attention of researchers from different fields (see e.g the November 1997 special issue of IEEE Transactions on Circuits and Systems, Part I). Various definitions of this phenomenon coexist and below we define synchronization following [2].

Consider $k$ dynamical systems described by the $k$ interconnected systems of ordinary differential equations:

$$
S_{i}: \quad \dot{x}_{i}=F_{i}\left(x_{1}, x_{2}, \ldots, x_{k}, t\right), \quad i=1, \ldots, k
$$

where $F_{i}: \mathbb{R}^{n_{1}} \times \ldots \times \mathbb{R}^{n_{k}} \times \mathbb{R}_{+} \rightarrow \mathbb{R}^{n_{i}}$. In this paper for simplicity we will give a coordinate dependent definition of synchronization. A more general coordinate-free definition of the problem of controlled synchronization involves a coordinate-free definition of dynamical systems and can be found for example in [2]. Associated with the systems (13) consider some time-dependent functional $Q$ defined on the solutions $x_{i}(\cdot)$ to these systems: $Q$ : $\mathcal{X}_{1} \times \ldots \times \mathcal{X}_{k} \times \mathbb{R}_{+} \rightarrow \mathbb{R}$, where $\mathcal{X}_{i} \subset\left\{x_{i}: \mathbb{R}_{+} \rightarrow \mathbb{R}^{n_{i}}\right\}$. We will say that solutions of the systems $S_{i}$ are synchronized if the value of the functional $Q$ is identically zero for these solutions for all $t \geq 0$. 
Definition 2 Solutions $x_{1}(t), \ldots, x_{k}(t)$ of the systems $S_{1}, \ldots, S_{k}$ with initial conditions $x_{1}(0), \ldots, x_{k}(0)$ are called synchronized with respect to the functional $Q$ if

$$
Q\left(x_{1}(\cdot), \ldots, x_{k}(\cdot), t\right) \equiv 0,
$$

for all $t \in \mathbb{R}_{+}$.

The solutions $x_{1}(t), \ldots, x_{k}(t)$ of the systems $S_{1}, \ldots, S_{k}$ with initial conditions $x_{1}(0), \ldots, x_{k}(0)$ are asymptotically synchronized with respect to the functional $Q$, if

$$
\lim _{t \rightarrow \infty}\left(Q\left(x_{1}(\cdot), \ldots, x_{k}(\cdot), t\right)\right)=0
$$

As one can notice this definition includes different variants of synchronization as introduced by several authors. For example, synchronization with respect to the functional

$$
Q\left(x_{1}(\cdot), \ldots, x_{k}(\cdot), t\right)=\sum_{i=2}^{k}\left|x_{1}(t)-x_{i}(t)\right|
$$

when all systems are identical and written in the same coordinate systems is usually referred to as identical synchronization. Obviously, here the functional $Q$ can be taken as a time independent function of the state variables. In this case the synchronization can be interpreted as a convergence to the "diagonal" set $\mathcal{A}=\left\{x_{1}, \ldots, x_{k} \in \mathbb{R}^{n}: x_{i}=x_{j} ; i, j=\right.$ $1, \ldots k\}$. If this is the case for all initial conditions in some open neighborhood of $\mathcal{A}$ then the synchronization is equivalent to attractivity of $\mathcal{A}$. For practical reasons it is convenient to consider a stronger case when the set $\mathcal{A}$ is not only attractive but also Lyapunov stable. This case is called strong synchronization [8]. However attractivity of $\mathcal{A}$ does not necessarily imply Lyapunov stability of $\mathcal{A}$. Indeed, even if $\mathcal{A}$ is a singleton, e.g. $\mathcal{A}=\{0\}$ then even if any solution of the system $\dot{x}=f(x), f(0)=0, x(t) \in \mathbb{R}^{n}, n \geq 2$ starting close to the origin satisfies $x(t) \rightarrow 0$ as $t \rightarrow \infty$ it does not mean that the origin is asymptotically stable, see [7]Section 40 . The case when $\mathcal{A}$ attracts solutions starting from a set of positive Lebesque measure but $\mathcal{A}$ is not Lyapunov stable is called weak synchronization [8]. Although the case of weak synchronization is of some theoretical interest, for practical purposes it is not so important because small disturbances affecting the system can destroy the synchronization. In the sequel, we will study strong synchronization, namely we will present sufficient conditions when $\mathcal{A}$ has a compact asymptotically stable subset.

Using the above definition of synchronization it is also possible to capture other synchronization notions. For example, the case $k=2$ and

$$
Q\left(x_{1}(\cdot), x_{2}(\cdot), t\right)=\left|x_{1}(t)-G\left(x_{2}(t)\right)\right|
$$

where $G$ is some continuous function corresponds to the so called generalized synchronization [14].

In what follows we are interested in the case of identical synchronization of identical systems forming a diffusive network.

In this case we rewrite the systems (11) in a form which can be obtained from (11) via a linear change of coordinates:

$$
\left\{\begin{array}{l}
\dot{z}_{j}=q\left(z_{j}, y_{j}\right) \\
\dot{y}_{j}=a\left(z_{j}, y_{j}\right)+C B u_{j}
\end{array}\right.
$$


where $z_{j}(t) \in \mathbb{R}^{n-m}, q: \mathbb{R}^{n-m} \times \mathbb{R}^{m} \rightarrow \mathbb{R}^{n-m}, a: \mathbb{R}^{n-m} \times \mathbb{R}^{m} \rightarrow \mathbb{R}^{m}$.

Let the eigenvalues of the matrix $\Gamma$ be ordered as: $0=\gamma_{1} \leq \gamma_{2} \leq \ldots \leq \gamma_{k}$.

Theorem 1 Assume that

A1. The functions $q$, a are locally Lipschitz continuous.

A2. The system

$$
\left\{\begin{array}{l}
\dot{z}=q(z, y) \\
\dot{y}=a(z, y)+C B u
\end{array}\right.
$$

is strictly semipassive with respect to the input $u$ and output $y$ with a radially unbounded storage function $V: \mathbb{R}^{n} \rightarrow \mathbb{R}_{+}$.

A3. There exist a $\mathcal{C}^{2}$-smooth positive definite function $V_{0}: \mathbb{R}^{n-m} \rightarrow \mathbb{R}_{+}$and a positive number $\alpha$ such that the following inequality is satisfied

$$
\left(\nabla V_{0}\left(z_{1}-z_{2}\right)\right)^{\top}\left(q\left(z_{1}, y_{1}\right)-q\left(z_{2}, y_{1}\right)\right) \leq-\alpha\left|z_{1}-z_{2}\right|^{2}
$$

for all $z_{1}, z_{2} \in \mathbb{R}^{n-m}, y_{1} \in \mathbb{R}^{m}$.

Then for all positive semidefinite $\Gamma$ as in (7) all solutions of the closed loop system (11), (12) are ultimately bounded and there exists $\bar{\gamma}$ such that for all positive semidefinite $\Gamma$ for which $\gamma_{2}>\bar{\gamma}$ there exists a globally asymptotically stable compact subset of the diagonal set $\mathcal{A}=\left\{x_{j}=\mathbb{R}^{n}: x_{i}=x_{j}, i, j=1, \ldots, k\right\}$.

Proof: Assumption A1 ensures existence of unique solutions of the closed loop system at least on a finite time interval. By Corollary 1 all solutions are ultimately bounded. Moreover, from the proof in [15], [16] one can see that the bounds in the inequalities $|z| \leq \mathcal{B}_{z},|y| \leq \mathcal{B}_{y}$ do not depend on the coupling matrix $\Gamma$.

Let $x=\operatorname{col}\left(x_{1}, x_{2}, \ldots, x_{k}\right) \in \mathbb{R}^{k n}, z=\operatorname{col}\left(z_{1}, z_{2}, \ldots, z_{k}\right) \in \mathbb{R}^{k(n-m)}, y=\operatorname{col}\left(y_{1}, y_{2}, \ldots, y_{k}\right) \in$ $\mathbb{R}^{k m}$. Introduce a new set of variables: $\tilde{x}_{1}=x_{1}, \tilde{x}_{2}=x_{1}-x_{2}, \ldots, \tilde{x}_{k}=x_{1}-x_{k}$. In matrix notation this change of coordinates can be written as $\tilde{x}=M x$, where $M=P \otimes I_{n}$ is the orthogonal matrix $k n \times k n$ with

$$
P=\left(\begin{array}{cc}
1 & 0 \\
1 & I_{k-1}
\end{array}\right)
$$

Notice that

$$
P \Gamma P^{-1}=\left(\begin{array}{cc}
0 & * \\
0 & \Gamma_{1}
\end{array}\right)
$$

where the $(k-1) \times(k-1)$ matrix $\Gamma_{1}$ has eigenvalues $\gamma_{2}, \ldots, \gamma_{k}$.

Denote $w_{1}=\operatorname{col}\left(\tilde{z}_{2}, \ldots, \tilde{z}_{k}\right)$ and $w_{2}=\operatorname{col}\left(\tilde{y}_{2}, \ldots, \tilde{y}_{k}\right)$. Assumption A3 implies the existence of a positive definite function $V_{1}: \mathbb{R}^{(k-1)(n-m)} \rightarrow \mathbb{R}_{+}$such that

$$
\left.\dot{V}_{1}\left(w_{1}, w_{2}\right)\right|_{w 2=0} \leq-\alpha_{1}\left|w_{1}\right|^{2}
$$

with $\alpha_{1}>0$.

Since $\Gamma=\Gamma^{\top}$ one can conclude that there exists a nonsingular $(k-1) \times(k-1)$ matrix $F$ such that $F \Gamma_{1} F^{-1}=\operatorname{diag}\left(\gamma_{2}, \ldots, \gamma_{k}\right)$. Introduce new coordinates $\tilde{w}_{1}=\left(F \otimes I_{n-m}\right) w_{1}$ 
and $\tilde{w}_{2}=\left(F \otimes I_{m}\right) w_{2}$. Since stability is invariant under a linear change of coordinates there exists a positive definite function $V_{2}: \mathbb{R}^{(k-1)(n-m)} \rightarrow \mathbb{R}_{+}$such that

$$
\left.\dot{V}_{2}\left(\tilde{w}_{1}, \tilde{w}_{2}\right)\right|_{\tilde{w}_{2}=0} \leq-\alpha_{2}\left|\tilde{w}_{1}\right|^{2}
$$

for some $\alpha_{2}>0$

Now consider the following Lyapunov function candidate:

$$
V_{3}\left(\tilde{w}_{1}, \tilde{w}_{2}\right)=V_{2}\left(\tilde{w}_{1}\right)+\tilde{w}_{2}^{\top} \tilde{w}_{2} / 2 \text {. }
$$

Notice that due to the ultimate boundedness of all solutions and smoothness of the right hand side of the closed loop system we have for $|z| \leq \mathcal{B}_{z},|y| \leq \mathcal{B}_{y}$

$$
\dot{V}_{2}\left(\tilde{w}_{1}, \tilde{w}_{2}\right)-\dot{V}_{2}\left(\tilde{w}_{1}, 0\right) \leq C_{1}\left|\tilde{w}_{1}\right| \cdot\left|\tilde{w}_{2}\right|
$$

and

$$
\frac{d}{d t} \tilde{w}_{2}^{\top} \tilde{w}_{2} / 2 \leq C_{2}\left|\tilde{w}_{1}\right| \cdot\left|\tilde{w}_{2}\right|-\left(\gamma_{2} \beta-C_{3}\right)\left|\tilde{w}_{2}\right|^{2}
$$

for some nonnegative $C_{1}, C_{2}, C_{3}$ with $\beta>0$ being the smallest eigenvalue of the matrix $C B$.

Hence

$$
\dot{V}_{3} \leq-\alpha\left|\tilde{w}_{1}\right|^{2}+\left(C_{1}+C_{2}\right)\left|\tilde{w}_{1}\right| \cdot\left|\tilde{w}_{2}\right|-\left(\gamma_{2} \beta-C_{3}\right)\left|\tilde{w}_{2}\right|^{2}
$$

In other words, for sufficienly large $\gamma_{2}$ we have for some $\varepsilon>0$

$$
\dot{V}_{3} \leq-\varepsilon\left(\left|\tilde{w}_{1}\right|^{2}+\left|\tilde{w}_{2}\right|^{2}\right)
$$

Integrating this inequality over $[0, t)$ yields

$$
V_{3}\left(\tilde{w}_{1}(t), \tilde{w}_{2}(t)\right) \leq V_{3}\left(\tilde{w}_{1}(0), \tilde{w}_{2}(0)\right)
$$

which proves the Lyapunov stability of the set $\tilde{w}_{1}=0, \tilde{w}_{2}=0$. Next we prove that this set contains a compact attractive subset which attracts all solutions.

Integrating $(20)$ over $[0, \infty)$ (recall that we have proved that all solutions are bounded and therefore exist on the infinite time interval) yields

$$
V_{3}\left(\tilde{w}_{1}(0), \tilde{w}_{2}(0)\right) \geq \varepsilon \int_{0}^{\infty}\left(\left|\tilde{w}_{1}(t)\right|^{2}+\left|\tilde{w}_{2}(t)\right|^{2}\right) d t
$$

The left hand side of this inequality is bounded, the integrand is nonnegative, therefore the integral exists and is finite. Consequently, all solutions of the closed loop system are bounded, the right hand side of the closed loop system is locally Lipschitz continuous, therefore the right hand side of the closed loop system is bounded for any solution, or, equivalently, $\dot{\tilde{w}}_{1}(t), \dot{\tilde{w}}_{2}(t)$ are bounded. Hence $\tilde{w}_{1}(t), \tilde{w}_{2}(t)$ are uniformly continuous in $t$ and therefore $\left|\tilde{w}_{1}(t)\right|^{2}+\left|\tilde{w}_{2}(t)\right|^{2}$ is uniformly continuous in $t$ as well. So, we have proved that there exists a finite integral over the infinite interval of the uniformly continuous function $\left|\tilde{w}_{1}(t)\right|^{2}+\left|\tilde{w}_{2}(t)\right|^{2}$. According to Barbalat's lemma [18] this function tends to zero, that is $\left|\tilde{w}_{1}(t)\right|^{2}+\left|\tilde{w}_{2}(t)\right|^{2} \rightarrow 0$ as $t \rightarrow \infty$. 
Remark 1 Since Assumption A2 implies ultimate boundedness of all solutions it is sufficient to require that Assumption $\mathrm{A} 3$ is valid only on the compact set $|z| \leq \mathcal{B}_{z},|y| \leq \mathcal{B}_{y}$. $\triangle$

At this point it is useful to make some comments. Consider the systems (17). It can be seen that these systems have inherent dynamics consistent with the external constraints $y_{1}-y_{2}=0, y_{1}-y_{3}=0, \ldots, y_{1}-y_{k}=0$ governed by the following equations:

$$
\begin{aligned}
& \left\{\begin{array}{l}
\dot{z}_{1}=q\left(z_{1}, y_{1}\right) \\
\vdots \\
\dot{z}_{k}=q\left(z_{k}, y_{1}\right)
\end{array}\right. \\
& \dot{y}_{1}=a\left(z_{1}, y_{1}\right)
\end{aligned}
$$

Moreover Assumption A3 can be interpreted as follows: the dynamics (21) driven by an admissible $y_{1}(t)$ has a noncritically stable set $z_{1}=z_{2}=\ldots=z_{k}$. Therefore Assumption A 3 is a natural generalization of the notion of hyperbolically minimum phaseness to the case of stabilization of sets.

Recall that hyperbolically minimum phaseness of each subsystem means that the system

$$
\dot{z}=q(z, 0)
$$

has a noncritically stable zero solution. As one can notice Assumption A3 is a sufficient condition for hyperbolic minimum phaseness but not necessary. A possible characterization of Assumption A3 can be given by the use of concept of convergent systems.

Consider the following system:

$$
\dot{z}=q(z, d)
$$

where $z(t) \in \mathbb{R}^{s}, d(t) \in \mathbb{D}, \mathbb{D}$ is some compact subset of $\mathbb{R}^{p}$, the function $d: \mathbb{R}^{1} \rightarrow \mathbb{D}$ is assumed to be continuous and the vector field $q: \mathbb{R}^{s} \times \mathbb{D} \rightarrow \mathbb{R}^{s}$ is locally Lipschitz continuous in $z$ and continuous in $d$.

Following Demidovich [5] we give the following definition:

Definition 3 The system (23) is said to be convergent if

$i$. all solutions $z(t)$ are well defined for all $t \in \mathbb{R}^{1}$ and all initial conditions $z(0)$.

ii. there exists a globally asymptotically stable unique solution $\bar{z}(t)$ bounded for all $-\infty<$ $t<\infty$, i.e. for any solution $z(t)$ it follows that

$$
\lim _{t \rightarrow \infty}|z(t)-\bar{z}(t)|=0
$$

Moreover, if $\left|z\left(t_{0}\right)-\bar{z}\left(t_{0}\right)\right|:=\delta\left(t_{0}\right)$ is sufficiently small so we have $|z(t)-\bar{z}(t)| \leq$ $C \exp \left(-\alpha\left(t-t_{0}\right)\right)$ with $C>0$ and $\alpha>0$, perhaps depending on $\delta\left(t_{0}\right)$, we will say that the system (23) is noncritically convergent.

If, additionally, the system (23) is convergent for all continuous functions $d$ from the given class $\mathcal{D}=\left\{d \in \mathcal{C}^{0}: \mathbb{R}^{1} \rightarrow \mathbb{D}\right\}$, the system (23) is referred to as convergent in $\mathcal{D}$.

According to [5] there exists a simple sufficient condition which guarantees that the system (23) is convergent (see [5], page 286; we present a more general result which can be derived from [5] via linear coordinate change): 
Theorem 2 Assume that there exists a positive definite matrix $P=P^{\top}>0$ such that all eigenvalues of the symmetric matrix

$$
\frac{1}{2}\left[P\left(\frac{\partial q}{\partial z}(z, \zeta)\right)+\left(\frac{\partial q}{\partial z}(z, \zeta)\right)^{\top} P\right]
$$

are negative for all $z \in \mathbb{R}^{s}$ and $\zeta \in \mathbb{D}$.

Then the system (23) is noncritically convergent in the class $\mathcal{D}$.

Remark 2 It is also worth mentioning that the result of Theorem 1 can be considered in the framework of passivity/passifiability with respect to sets. Indeed, consider the systems (17) in closed loop with

$$
u_{j}=-\gamma_{j 1}\left(y_{j}-y_{1}\right)-\gamma_{j 2}\left(y_{j}-y_{2}\right)-\ldots-\gamma_{j k}\left(y_{j}-y_{k}\right)+v_{j}
$$

where $j=1, \ldots, k$ and $v=\operatorname{col}\left(v_{1}, \ldots, v_{k}\right)$ is a new input. In this case Theorem 1 ensures existence of a $(k-1) m \times k m$ matrix $L$ such that the closed loop system is strictly passive with respect to set $\mathcal{A}$ with input $L v$ and output $\tilde{w}_{2}$ with a storage function being the function $V_{3}$ as in equation (19).

\section{Example 1 Synchronization of an array of Lorenz systems.}

Consider the following $k$ systems:

$$
\left\{\begin{array}{l}
\dot{x}_{j}=\sigma\left(y_{j}-x_{j}\right)+u_{j} \\
\dot{y}_{j}=r x_{j}-y_{j}-x_{j} z_{j} \\
\dot{z}_{j}=-b z_{j}+x_{j} y_{j}
\end{array}\right.
$$

with $j=1, \ldots, k$ and

$$
u_{j}=-\gamma_{j 1}\left(y_{j}-y_{1}\right)-\gamma_{j 2}\left(y_{j}-y_{2}\right)-\ldots-\gamma_{j k}\left(y_{j}-y_{k}\right)
$$

We will show that if the smallest nonzero eigenvalue of the matrix $\Gamma$ is large enough then the $k$ systems synchronize according to

$$
\sum_{j=2}^{k}\left|x_{1}(t)-x_{j}(t)\right| \rightarrow 0, \quad \sum_{j=2}^{k}\left|y_{1}(t)-y_{j}(t)\right| \rightarrow 0, \quad \sum_{j=2}^{k}\left|z_{1}(t)-z_{j}(t)\right| \rightarrow 0
$$

as $t \rightarrow \infty$.

First we check that the system

$$
\left\{\begin{array}{l}
\dot{x}_{1}=\sigma\left(y_{1}-x_{1}\right)+u \\
\dot{y}_{1}=r x_{1}-y_{1}-x_{1} z_{1} \\
\dot{z}_{1}=-b z_{1}+x_{1} y_{1}
\end{array}\right.
$$

is strictly semipassive with respect to the input $u$ and output $x_{1}$. To this end consider the smooth function

$$
V\left(x_{1}, y_{1}, z_{1}\right)=\frac{1}{2}\left(x_{1}^{2}+y_{1}^{2}+\left(z_{1}-\sigma-r\right)^{2}\right)
$$


Its time derivative with respect to the uncontrolled system $(u(t) \equiv 0)$ satisfies:

$$
\dot{V}\left(x_{1}, y_{1}, z_{1}\right)=-\sigma x_{1}^{2}-y_{1}^{2}-b\left(z_{1}-\frac{\sigma+r}{2}\right)^{2}+b \frac{(\sigma+r)^{2}}{4}
$$

It is seen that $\dot{V}=0$ determines an ellipsoid outside which the derivative of $V$ is negative. If $K$ satisfies

$$
K^{2}=\frac{1}{4}+\frac{b}{4} \max \left\{\frac{1}{\sigma}, 1\right\}
$$

then this ellipsoid lies inside the ball

$$
\Xi=\left\{x, y, z: x^{2}+y^{2}+(z-\sigma-r)^{2} \leq K^{2}(\sigma+r)^{2}\right\}
$$

which means that all solutions of the uncontrolled system approach within some finite time the set defined by (28). Additionally, it is obvious that $(\nabla V)^{\top} g=x_{1}$. Therefore the function $V$ is a storage function which proves strict semipassivity of the system (26).

Secondly we should find the zero dynamics imposing the external constraints $x_{1}=$ $x_{j}, j=1, \ldots, k$ :

$$
\left\{\begin{array}{l}
\dot{y}_{1}=r x_{1}-y_{1}-x_{1} z_{1} \\
\dot{z}_{1}=-b z_{1}+x_{1} y_{1} \\
\dot{y}_{2}=r x_{1}-y_{2}-x_{1} z_{2} \\
\dot{z}_{2}=-b z_{2}+x_{1} y_{2} \\
\vdots \\
\dot{y}_{k}=r x_{1}-y_{k}-x_{1} z_{k} \\
\dot{z}_{k}=-b z_{k}+x_{1} y_{k} \\
\dot{x}_{1}=\sigma\left(y_{1}-x_{1}\right)
\end{array}\right.
$$

Now let us show that the system

$$
\left\{\begin{array}{l}
\dot{y}_{1}=r x_{1}-y_{1}-x_{1} z_{1} \\
\dot{z}_{1}=-b z_{1}+x_{1} y_{1}
\end{array}\right.
$$

is noncritically convergent for any bounded $x_{1}(t)$. Indeed, the symmetrized Jacobi matrix for this system has two eigenvalues -1 and $-b$ and therefore, according to Theorem 2 there exists a quadratic function which satisfies Assumption A3 of Theorem 1.

Thus all the conditions of Theorem 1 are satisfied and so there exists a number $\bar{\gamma}$ such that for sufficiently large $\gamma_{2}>\bar{\gamma}$ the system of $k$ diffusively coupled Lorenz systems has an asymptotically stable compact subset of the set $\left\{x_{1}=x_{2}=\ldots=x_{k}, y_{1}=y_{2}=\ldots=\right.$ $\left.y_{k}, z_{1}=z_{2}=\ldots=z_{k}\right\}$.

\section{On diffusion driven instability}

In the previous section we considered a phenomenon which can be observed in a network of diffusively coupled minimum phase systems. Even in case when each separate free system oscillates irregularly coupled together they may exhibit some kind of synchronization. In 
this case synchronization can be considered as a sign of cooperation via diffusion. However arrays of diffusively coupled systems may exhibit asynchronous oscillatory behavior also as a result of cooperation via diffusion. Namely, assume that each free system in the array of diffusively coupled systems is globally asymptotically stable. A common understading of diffusion is a smoothening or trivializing process; however an array of diffusively coupled globally asymptotically stable systems may demonstrate oscillatory behavior.

First we restrict the class of oscillatory systems we will deal with. Given a system of autonomous differential equations

$$
\dot{x}=F(x)
$$

where $x \in \mathbb{R}^{n}$. We will say that the system (30) is oscillatory in the sense of Yakubovich, or $Y$-oscillatory if any solution of (30) is bounded and for almost all initial conditions the corresponding solution does not tend to a constant (see e.g. [24]).

The purpose of this section is to give an explicit construction of diffusively coupled globally asymptotically stable systems that become oscillatory being interconnected. One of the possible motivation of this problem lies in the field of mathematical biology, see [20].

We assume that the topology of the interconnection is described by the matrix $\Gamma$ as defined in (7) with entries $\gamma_{i j}$ as in Definition 1.

Let $A$ be a $n \times n$ matrix, $n \geq 3$

$$
A=\left(\begin{array}{ll}
A_{11} & A_{12} \\
A_{21} & A_{22}
\end{array}\right)
$$

where $A_{11}$ is a $(n-m) \times(n-m)$ matrix, $1 \leq m \leq n-2$ and the other matrices are of corresponding dimensions. Let $B$ and $C$ be full rank $n \times m$ and $m \times n$ matrices such that the product $C B$ is a positive definite matrix.

The following theorem was proved in [16].

Theorem 3 Assume that the following assumptions hold for the above matrices $A, B$ and $C$.

A1 The matrix $A$ is Hurwitz.

A2 The matrix $A_{11}$ has an even nonzero number of eigenvalues with positive real parts.

A3 The matrix $(C B)^{-1} T$, where $T=\left(A_{22}-A_{21} A_{11}^{-1} A_{12}\right)$, has no positive real eigenvalues. Then there exists a $\mathcal{C}^{1}$-function $f: \mathbb{R}^{n} \rightarrow \mathbb{R}^{n}, f(z, y)=\operatorname{col}(q(z, y), a(z, y))$, where $z \in \mathbb{R}^{n-m}, y \in \mathbb{R}^{m}, q: \mathbb{R}^{n-m} \times \mathbb{R}^{m} \rightarrow \mathbb{R}^{n-m}, a: \mathbb{R}^{n-m} \times \mathbb{R}^{m} \rightarrow \mathbb{R}^{m}$, such that

$$
A_{11}=\frac{\partial q}{\partial z}(0,0), \quad A_{12}=\frac{\partial q}{\partial y}(0,0), \quad A_{21}=\frac{\partial a}{\partial z}(0,0), \quad A_{11}=\frac{\partial a}{\partial y}(0,0)
$$

Moreover we have that

1) The system

$$
\left\{\begin{array}{l}
\dot{z}=q(z, y) \\
\dot{y}=a(z, y)
\end{array}\right.
$$

has a unique globally asymptotically stable equilibrium at the origin. 
2) For all $\gamma_{i j} \geq 0$ the system consisting of $k$ diffusively coupled systems

$$
\left\{\begin{array}{l}
\dot{z}_{j}=q\left(z_{j}, y_{j}\right) \\
\dot{y}_{j}=a\left(z_{j}, y_{j}\right)+C B u_{j} \\
u_{j}=-\gamma_{j 1}\left(y_{j}-y_{1}\right)-\gamma_{j 2}\left(y_{j}-y_{2}\right)-\ldots-\gamma_{j k}\left(y_{j}-y_{k}\right)
\end{array}\right.
$$

has ultimately bounded solutions, has the origin as a unique equilibrium and there exists a positive number $\bar{\gamma}$ such that for all $\gamma_{k}>\bar{\gamma}$, where $\gamma_{k}$ stands for the maximal eigenvalue of the matrix $\Gamma$ (cf. (7)), the closed loop system is Y-oscillatory.

Let us clarify the assumptions of the theorem. Assumption A1 allows to find a smooth vector field on $\mathbb{R}^{n}$ such that $A$ is the Jacobian of $f$ at zero and therefore the origin is a noncritical locally stable equilibrium of the system $\dot{x}=f(x)$. Assumption A2 guarantees that the zero dynamics of the system $\dot{x}=f(x)+B u, \quad y=C x$ is hyperbolically unstable at the origin. Assumption A3 is required in order to prove that when losing stability the origin does not undergo a bifurcation that results in the birth of additional equilibria. It is worth to mention that Assumption A3 cannot be satisfied if the matrix $A_{11}$ has an odd number of eigenvalues with positive real parts (This follows from Schur's decomposition since Assumption A3 in this case contradicts the stability of the matrix $A$ ).

The idea of the proof is relatively easy. It is based on an explicit construction of the vector field $f$ in the form $f(x)=A\left(1+|x|^{2}\right) x$. In this case stability of the matrix $A$ allows one to prove ultimate boundedness of solutions of the interconnected systems (the result follows from Lemma 1). According to Gerschgorin's theorem the matrix $\Gamma$ has nonnegative eigenvalues, therefore the diffusive feedback can be understood as a kind of "negative" feedback which is a "destabilizing" feedback for nonminimum phase systems. Moreover the hypotheses imply that the closed loop system has a unique equilibrium which, for sufficiently large $\gamma_{k}$, is hyperbolically unstable and therefore according to the HartmanGrobman theorem the set of initial conditions for which the corresponding solution tends to a constant is of zero measure. Details of the proof can be found in [16].

It is worth mentioning that the condition $n \geq 3$ is necessary in the following sense: if for $n=2$ the closed loop system is Y-oscillatory then each free system cannot be globally asymptotically stable (see Proposition 1 in [16]). This result answers one of the questions posed by Smale in [20] of finding a minimal order example of globally asymptotically stable systems which become oscillatory via diffusion.

Example 2 Consider the following $k$ systems

$$
\left\{\begin{array}{l}
\dot{x}_{1}=A x_{1}\left(1+\left|x_{1}\right|^{2}\right)+B u_{1} \\
\dot{x}_{2}=A x_{2}\left(1+\left|x_{2}\right|^{2}\right)+B u_{2} \\
\vdots \\
\dot{x}_{k}=A x_{k}\left(1+\left|x_{k}\right|^{2}\right)+B u_{k}
\end{array}\right.
$$

where $x_{j} \in \mathbb{R}^{3}, y_{j}=C x_{j}$,

$$
u_{j}=-\gamma_{j 1}\left(y_{j}-y_{1}\right)-\gamma_{j 2}\left(y_{j}-y_{2}\right)-\ldots-\gamma_{j k}\left(y_{j}-y_{k}\right)
$$


and

$$
A=\left(\begin{array}{rrr}
1 & -1 & 1 \\
1 & 0 & 0 \\
-4 & 2 & -3
\end{array}\right), \quad B=\left(\begin{array}{l}
0 \\
0 \\
1
\end{array}\right), \quad C=\left(\begin{array}{lll}
0 & 0 & 1
\end{array}\right)
$$

Simple calculations show that all conditions of Theorem 3 are satisfied and therefore for sufficiently large $\gamma_{k}$, where $\gamma_{k}$ is the largest eigenvalue of the matrix $\Gamma$ the closed loop system is $\mathrm{Y}$-oscillatory.

The above results allow for a better understanding of different oscillatory phenomena occuring as a result of cooperation via diffusion. Loosely speaking, in an array of diffusively coupled minimum phase systems one can expect the existence of synchronous properties while, in contrast, an array of diffusively coupled nonminimum phase systems may exhibit oscillatory behavior even when each free system is globally asymptotically stable.

\section{$7 \quad$ Properties of diffusive networks}

In the previous sections we discussed some oscillatory phenomena occuring as a result of diffusive interaction between identical subsystems. We presented results which are essentially based on some properties of the matrix $\Gamma$ which describes the topology of the interconnections. In this section we will discuss the synchronization or oscillatory behavior of the diffusively coupled systems in relation to the topology of the coupling.

Definition 4 A system consisting of $k$ diffusively coupled systems is said to be a cellular diffusive network if it cannot be decomposed in two or more disconnected subsystems.

First we give a simple characterization of diffusive networks.

Lemma 2 A system consisting of $k$ diffusively coupled systems is a cellular diffusive network if and only if any $p \times p, p \leq k-1$ principal submatrix of $\Gamma$ is nonsingular.

Proof: If the coupled system can be decomposed in disconnected systems then obviously there exists a singular principal submatrix of $\Gamma$ of the order less than $k$. Therefore we need to prove that if there is a $p \times p, p \leq k-1$ singular principal submatrix of $\Gamma$ then the whole system can be decomposed in at least two sets of mutually not interconnected systems. In other words, if there is a singular principal submatrix of $\Gamma$ of the order strictly less than $k$ then necessarily $\Gamma$ has at least two zero eigenvalues. Consider the matrix

$$
\Gamma_{\varepsilon}=\left(\begin{array}{cc}
\Gamma^{\prime} & \varepsilon a \\
\varepsilon a^{\top} & \varepsilon \Gamma^{\prime \prime}
\end{array}\right)
$$

where $\varepsilon \in[0,1]$ and $\Gamma^{\prime}$ is a $p \times p, 1 \leq p \leq k-1$ principal submatrix of $\Gamma$. By assumption, $\Gamma^{\prime}$ is singular, that is $\Gamma_{0}$ has two zero eigenvalues. Consider the matrix

$$
\left(\begin{array}{cc}
0_{p} & \varepsilon a \\
\varepsilon a^{\top} & \varepsilon \Gamma^{\prime \prime}
\end{array}\right)
$$


It has only nonnegative eigenvalues. Therefore according to Weyl's perturbation theorem the zero eigenvalues of the perturbed matrix cannot decrease and the number of zero eigenvalues of $\Gamma_{1}$ can not be less than the number of zero eigenvalues of $\Gamma_{0}$.

Definition 5 The maximal number $N$ of cells connected to one cell in a diffusively coupled array of systems is called the density of the cellular network.

Definition 6 A cellular diffusive network is said to be regular if

1. All coupling constants are equal: $\gamma_{i j}=\gamma$ for all $i \neq j$

2. Each cell is connected to $N$ other cells.

Notice that we did not impose any other restrictions on the topology of the interconnections, for example symmetry. Note that regular networks can model very complex structures including isotropic or anisotropic media. It is worth mentioning, however, that some symmetry in the coupling can generate very interesting properties of solutions bifurcated via a Poincaré-Andronov-Hopf bifurcation which leads to oscillations in coupled systems [6]. Moreover in some particular cases, e.g. for linear one-dimensional arrays, for rosette-like structures, the matrix $\Gamma$ has a special structure (it turns out to be a cyclic matrix) for which all eigenvalues can be found analytically [9].

It is worth mentioning that the numbers $k$ (i.e. number of cells) and $N$ (i.e. maximal number of connections at each cell) in no way define the complete topology of the network. Many different structures of the network can correspond to the same $k$ and $N$. As before denote the eigenvalues of the matrix $\Gamma$ as follows: $0=\gamma_{1} \leq \gamma_{2} \leq \ldots \leq \gamma_{k}$. For any given $k$ and $N$ the largest possible $\gamma_{2}$ will be denoted as $\gamma_{2}(k, N)$ while the smallest possible $\gamma_{k}$ will be denoted as $\gamma_{k}(k, N)$.

As we have seen in the previous sections stability analysis in diffusive networks essentially depends on two eigenvalues of the matrix $\Gamma$ which describes the topology of the interconnections. Therefore in the design of diffusive networks the following discrete optimization problems are of interest. Given $N$ and $k$. Find a structure of the (regular) network which maximizes $\gamma_{2}(k, N)$ (maximizes $\gamma_{k}(k, N)$ ) under the constraint that all nonzero $\gamma_{i j}, i \neq j$ are bounded from below and above by given constants. In general an analytic solution to these problems is an open question. However, using methods of discrete programming one can find solutions based on computer computation. Clearly, the computational complexity increases significantly for large $k$. In what follows we will present a solutions for particular cases and then we will focus our attention to asymptotic behavior of $\gamma_{2}(k, N)$ and $\gamma_{k}(k, N)$ when $k$ tends to infinity.

Example 3 Consider the following problem. Given the density $N$, find $k$ and a structure for a regular network such that $\gamma_{2}(k, N)$ is maximal possible for all $k$. A solution to this problem is trivial: $k=N+1$. It corresponds to the "all to all" structure. In this case the matrix $\Gamma$ has $N$ eigenvalues equal to $(N+1) \gamma$ and one zero eigenvalue. Since $\operatorname{tr} \Gamma=N(N+1) \gamma$ the solution is optimal. 
Example 4 Now consider a similar problem. Given $N$, find $k$ and a structure of regular network such that $\gamma_{k}(k, N)$ is maximal possible for all $k$. A solution is $k=2 N$. Take two clusters consisting of $N$ cells and connect each cell from the first cluster to each cell from the second cluster. The matrix $\Gamma$ in this case has one zero eigenvalue, one eigenvalue equal to $2 N \gamma$ and $2(N-1)$ eigenvalues equal to $N \gamma$. According to Gerschgorin's theorem $2 N \gamma$ is the maximal possible eigenvalue for regular networks of density $N$ for arbitrary $k$. Therefore the solution is optimal.

Next we will investigate the asymptotic behavior of $\gamma_{2}(k, N)$ and $\gamma_{k}(k, N)$ when $k \rightarrow \infty$. We are able to establish two remarkable facts for regular networks.

Theorem 4 For regular networks the following relation is valid

$$
\lim _{k \rightarrow \infty} \gamma_{2}(k, N)=0
$$

Proof: For simplicity take $\gamma=1$. Rewrite the matrix $\Gamma$ in the following form

$$
\Gamma=\left(\begin{array}{cc}
\Gamma^{\prime} & a \\
a^{\top} & N
\end{array}\right)
$$

where the column vector $a \in \mathbb{R}^{k-1}$ contains only $N$ entries equal to -1 . According to Gerschgorin's theorem all eigenvalues of $\Gamma^{\prime}$ are nonnegative. According to Lemma 2 $\Gamma^{\prime}$ is nonsingular, that is $\Gamma^{\prime}$ is positive definite. Consider the vector $z \in \mathbb{R}^{k-1}, z=$ $(1 / \sqrt{k-1}, 1 / \sqrt{k-1}, \ldots, 1 / \sqrt{k-1})$. Clearly, $|z|=1$. Moreover it follows that $z^{\top} \Gamma^{\prime} z=$ $N(k-1)^{-1}$ (the sum of $k-N-1$ rows of $\Gamma^{\prime}$ is zero, while the sum of the other rows is 1 ). According to Fischer's theorem (see, e.g. [21]) the smallest eigenvalue of the symmetric matrix $\Gamma^{\prime}$ is equal to $\min _{|z|=1} z^{\top} \Gamma^{\prime} z$. Therefore the smallest eigenvalue of $\Gamma^{\prime}$ is less or equal to $N(k-1)^{-1}$ and it tends to zero when $k$ tends to $\infty$.

Notice that

$$
M \Gamma M^{\top}=\left(\begin{array}{cc}
\Gamma^{\prime} & 0 \\
0 & N-a^{\top} \Gamma^{\prime-1} a
\end{array}\right)
$$

where

$$
M=\left(\begin{array}{cc}
I_{k-1} & 0 \\
-a^{\top} \Gamma^{\prime-1} & 1
\end{array}\right)
$$

Since a congruence transformation preserves the inertia (Silvester-Jacobi theorem) and $\Gamma^{\prime}$ is nonsingular while $\Gamma$ is singular it follows that $a^{\top} \Gamma^{\prime-1} a=N$. Moreover since $\Gamma^{\prime-1}$ is positive definite and the vector $a$ consists of $N$ nonzero entries one can see that the last string of $M$ consists of $N+1$ nonzero entries which are bounded for all $k$. Rewrite the congruence transformation in the following form

$$
M \Gamma=\left(\begin{array}{cc}
\Gamma^{\prime} & 0 \\
0 & 0
\end{array}\right)\left(M^{-1}\right)^{\top}
$$

where

$$
\left(M^{-1}\right)^{\top}=\left(\begin{array}{cc}
I_{k-1} & \Gamma^{\prime-1} a \\
0 & 1
\end{array}\right)
$$


Since the eigenvalues of $\Gamma^{\prime}$ can be made arbitrary small for large enough $k$ for any $\varepsilon>0$ it is possible to find $\bar{k}$ and a two-dimensional subspace $\mathbb{D}$ of $\mathbb{R}^{k}$ such that $\left|\operatorname{diag}\left\{\Gamma^{\prime}, 0\right\} z\right|<\varepsilon$ for all $z \in \mathbb{D}$ with $|z|=1$ and all $k \geq \bar{k}$. Using properties of the matrix $M$ one can see that this implies the existence of a two-dimensional subspace $\mathbb{D}_{1}$ of $\mathbb{R}^{k}$ such that $|\Gamma z|$ can be made arbitrary small for large enough $k$ and $z \in \mathbb{D}_{1},|z|=1$. According to Fischer's theorem

$$
\gamma_{2}=\min _{\operatorname{dim} \mathcal{X}=2} \max _{z \in \mathcal{X},|z|=1} z^{\top} \Gamma z
$$

Therefore

$$
\gamma_{2} \leq \max _{z \in \mathbb{D}_{1},|z|=1} z^{\top} \Gamma z
$$

In orther words, $\gamma_{2}(k, N) \rightarrow 0$ as $k \rightarrow \infty$.

Remark 3 The theorem statement remains true for arbitrary cellular networks if we assume that its density and maximal value of the coupling constants $\gamma_{i j}, i \neq j$ are bounded for $k \rightarrow \infty$.

In other words, if the number of interconnections grows at most linearly with respect to the number of cells, then the smallest nonzero eigenvalue of the matrix $\Gamma$ which is "responsible" for synchronization with respect to the functional (16) decays as $k$ goes to infinity. This fact significantly restricts possible synchronous modes in large diffusive networks. For example, if the regular network consisting of Lorenz systems studied in Example 1 grows then for any given $\gamma$ and $N$ there exist a $\vec{k}$ such that there is no synchronization as soon as $k \geq \vec{k}$. This fact explains the computer simulations carried out in [12].

Moreover, using the same technique as in the proof of the previous theorem it is possible to show that zero is an accumulation point in the spectrum of the matrix $\Gamma$ when $k$ increases. At the same time if one allows for the number of interconnections to grow quadratically with respect to the number of cells in regular networks (this is the case for example in "all to all' structures) then the smallest nonzero eigenvalue grows with $k$ and the coupling gain $\gamma$ which ensures synchronization decays as $k^{-1}$ (see Proposition 1 in [15]).

Now let us investigate the asymptotic behavior of the largest eigenvalue of the matrix $\Gamma$ which is "responsible" for generation of oscillations in diffusive networks.

Theorem 5 For regular networks the following relation is valid:

$$
\lim _{k \rightarrow \infty} \gamma_{k}(k, N)=2 N \gamma
$$

Proof: For simplicity take $\gamma=1$. According to the Gerschgorin theorem all eigenvalues of $\Gamma$ are less or equal to $2 N$, that is the matrix $\Gamma_{+}=2 N I_{k}-\Gamma$ has no negative eigenvalues. If the matrix $\Gamma_{+}$is singular then the largest eigenvalue of $\Gamma$ is equal to $2 N$, therefore consider the case when $\Gamma_{+}$is nonsingular and therefore positive definite. Using the same technique as in the proof of the previous theorem we prove that the smallest eigenvalue of $\Gamma_{+}$tends to zero as as $k \rightarrow \infty$, in other words, the largest eigenvalue of $\Gamma$ tends to $2 N$. 
Example 5 This theorem allows us to establish that the statement conjectured by Wu and Chua [27] is wrong. Given two diffusive networks with coupling matrices $\Gamma^{\prime}$ and $\Gamma^{\prime \prime}$ such that $\gamma_{2}^{\prime}=\gamma_{2}^{\prime \prime}$. The Wu-Chua conjecture claims that the conditions of global (identical) synchronization for the first network are equivalent to the conditions of global synchronization in the second network. Theorem 1 gives sufficient conditions under which this statement is true. At the same time Theorem 5 asserts that for growing regular diffusive networks the largest eigenvalue does not tend to zero. Since it is possible to design a system consisting of two diffusively coupled subsystems which are synchronized only if the coupling strength lies in some region (e.g. when each system is hyperbolically nonminimum phase), in the general case the $\mathrm{Wu}$-Chua conjecture is not true.

\section{Conclusion}

In this paper we presented analytical tools for the study of oscillatory behavior in the arrays of diffusively coupled systems with an arbitrary topology of interconnections. The dynamics of the network is essentially based on stability property with respect to sets of the constrained dynamics usually referred to as zero dynamics. In case that this dynamics has a noncritically asymptotically stable compact set consistent with $x_{1}=x_{2}=\ldots=x_{k}$ the whole system has a tendency to synchronization. Instability of the zero dynamics, in turn, leads to generation of oscillations in diffusive networks.

We also have shown that in growing networks the growth rate of the number of interconnection is essential for synchronization but not so important for generation of oscillations via diffusion in large networks.

\section{Acknowledgements}

This work was supported in part by the INTAS Foundation under contract 94-0965, the RFBR Grant 96-01-01151, the Russian Federal Programme "Integration" (project 2.1-589).

\section{References}

[1] Belykh, V.N., N.N. Verichev, L. Kocarev and L.O. Chua, [1993] "On chaotic synchronization in a linear array of Chua's circuits," J. Circuits Syst. and Comput., vol. 3, pp. 579-589.

[2] Blekhman, I.I., A.L. Fradkov, H. Nijmeijer and A.Yu. Pogromsky [1997] "On selfsynchronization and controlled synchronization", Systems \& Control Letters, vol.31 pp. 299-306.

[3] Byrnes, C.I., A. Isidori and J.C. Willems [1991] "Passivity, feedback equivalence and the global stabilization of minimum phase nonlinear systems", IEEE Trans. Aut. Contr., vol. 36(11), pp. 1228-1240. 
[4] Chua, L.O., M. Hasler, G.S. Moschytz and J. Neirynck [1995] "Autonomous cellular neural networks: A unified paradigm for pattern formation and active wave propagation," IEEE Trans. Circ. Sys. I, vol. 42, pp. 559-577.

[5] Demidovich, B.P. [1967] Lectures on stability theory, Moscow-Nauka, (in Russian).

[6] Dionne, B., M. Golubitsky and I. Stewart, [1996] "Coupled cells with internal symmetry," I, II, Nonlinearity, vol. 9, pp. 559-599.

[7] Hahn, W. [1967] Stability of motion, Springer-Verlag, Berlin.

[8] Hasler M., and Y. Maistrenko, [1997], "An introduction to the synchronization of chaotic systems: coupled skew tent maps," IEEE Transactions Circ. Syst., Part I, vol. 44 , pp. $856-866$.

[9] Hoppensteadt, F.C. [1986] An introduction to the mathematics of neurons (Cambridge University press, Cambridge).

[10] Hill, D.J. and P.J. Moylan [1976] "The stability of nonlinear dissipative systems", IEEE Trans. Automat. Control, vol. 21, pp. 708-711.

[11] Lin, Y., E.D. Sontag and Y. Wang [1996] "A smooth converse Lyapunov theorem for robust stability", SIAM J. Control Optimization, vol. 34, pp. 124-160.

[12] Milanovic, V. and M. E. Zaghloul [1996] "Synchronization of chaotic neural networks and applications to communications," Int. J. Bifurcation Chaos, vol.6(12B), pp. 2571-2585.

[13] Ortega, R., A. Loria, P.J. Nicklasson and H. Sira-Ramirez [1998] Passivity-Based Control of Euler-Lagrange Systems, Springer-Verlag, London.

[14] Parlitz, U. and L. Kocarev [1998] "Synchronization of chaotic systems", in Handbook of Chaos Control (ed. H.G. Schuster) (Springer-Verlag).

[15] Pogromsky, A. Yu. [1998] "Passivity based design of synchronizing systems" Int. J. Bifurcation and Chaos, vol. 8 (2), pp. 295-319.

[16] Pogromsky A. Yu., T. Glad and H. Nijmeijer [1998] "On diffusion driven oscillations in coupled dynamical systems", Int. J. Bifurcation Chaos, to appear.

[17] Polushin I.G., Hill D.J., Fradkov A.L., [1998] "Strict quasipassivity and ultimate boundedness for nonlinear control systems," Proc. 4th IFAC Symposium on Nonlinear Control Systems, NOLCOS'98, Enschede, The Netherlands, pp.527-532.

[18] Popov, V.M. [1973] Hyperstability of control systems, New York: Springer-Verlag, (in Romanian: 1966).

[19] Setti, G., P. Thiran, and C. Serpico [1998] "An approach to information propagation in 1-D cellular neural networks - Part II: Global propagation," IEEE Trans. Circ. Sys. I, vol. 45, pp. 790-811.

[20] Smale, S. [1976] "A mathematical model of two cells via Turing's equation," pp. 354-367, in Marsden, J.E. and M. McCracken (eds.) [1976] The Hopf bifurcation and its applications, Springer-Verlag, New York.

[21] G.W. Stewart, Ji-Guang Sun, [1990] Matrix Perturbation Theory, Academic Press. 
[22] Thiran, P., K.R. Crounse, L.O. Chua and M. Hasler [1995] "Pattern formation properties of autonomous cellular neural networks," IEEE Trans. Circ. Sys. I, vol. 42, pp. 757-776.

[23] Thiran, P., G. Setti and M. Hasler [1998] "An approach to information propagation in 1-D cellular neural networks - Part I: Local diffusion," IEEE Trans. Circ. Sys. I, vol. 45, pp. 777-789,

[24] Tomberg, E.A. and V.A. Yakubovich [1989] "Self-oscillatory conditions in nonlinear systems", Siberian Math. Journal, v. 30(4), pp. 180-194, in Russian.

[25] Van der Schaft, A.J. $\mathcal{L}_{2}$-gain and passivity techniques in nonlinear control, Lecture Notes on Control and Information Sciences, Springer, London, 1996

[26] Wu, C.W. and L.O. Chua [1995] "Synchronization in an array of linearly coupled dynamical systems", IEEE Trans. Circuits Systems, I, vol.42, pp. 430-447.

[27] Wu, C.W. and L.O. Chua [1995] "On a conjecture regarding the synchronization in an array of linearly coupled dynamical systems", IEEE Trans. Circuits Systems, I, vol.43, pp. 161-165. 